\title{
Simposio: Carcinoma Gástrico
}

\author{
Gastric Cancer symposium
}

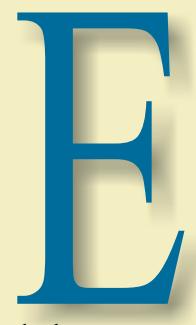

121 de enero de 2021 se llevó a cabo el Simposio sobre Carcinoma Gástrico organizado por la Peruvian American Medical Society (PAMS). Esta es una entidad caritativa formada en 1973 por médicos peruanos radicados en USA con dos objetivos: 1. Mejorar la salud médica de los peruanos más necesitados y, 2. Mejorar la educación médica en el Perú.

El Simposio fue coauspiciado por PAMS, la Universidad Peruana Cayetano Heredia, la Universidad Católica Santa María de Arequipa, el Colegio Médico del Perú y el Instituto Nacional de Enfermedades Neoplásicas (INEN) en Lima.

El carcinoma gástrico es uno de los cánceres más frecuentes y más letales en el Perú( ${ }^{(1)}$. Por consiguiente, la revisión de aspectos importantes de esta patología es significativa para la salud de los peruanos y, por ello, los manuscritos del Simposio son publicados en la revista DIAGNÓSTICO.

Las cuatro ponencias del Simposio dieron un enfoque multidisciplinario al tema incluyendo: a. Manifestaciones clínicas; b. Factores de riesgo - rol del Helicobacter pylori; c. Papel de la cirugía y, d. Papel de la quimioterapia. Las presentaciones y discusión de los temas se hicieron electrónicamente y fueron difundidas también por redes sociales a través del Perú y varios países de América Latina.

Se enfatizó la presentación tardía del cáncer gástrico en el Perú en un $75 \%$ de casos, incluyendo a síntomas como pérdida de peso, hemorragia digestiva, anemia y obstrucción del tracto digestivo ${ }^{(2)}$. A veces, se asocian síndromes paraneoplásticos como la caquexia, manifestaciones dermatológicas (acantosis nigricans, acroqueratosis, hipertricosis lanugosa, pénfigo) dermatomiositis, vasculitis, etc. ${ }^{(3)}$. La evaluación clínica del paciente incluye estudios de rutina de sangre y específicos como la endoscopia gástrica para el diagnóstico histológico del tumor. También, estudios de estadiaje radiológico como son la radiografía del tórax y la tomografía del abdomen y pelvis para descartar metástasis. El adenocarcinoma sigue siendo el cáncer gástrico más frecuente y a ello, ahora se agrega la clasificación molecular y la evaluación de marcadores tumorales como el Her - 2neu y el PD-1 que tienen potencial terapéutico para el paciente ${ }^{(4-6)}$.

Los factores de riesgo son múltiples e incluyen al sexo masculino, edad avanzada, factores nutricionales como exceso de sal, de nitritos y alimentos ahumados, poca ingesta de frutas y verduras y de vitaminas $\mathrm{A}$ y $\mathrm{C}$, tabaquismo, falta de refrigeración, infecciones como la del Helicobacter pylori y del virus Epstein Barr, bajo nivel socioeconómico y otros ${ }^{(7-9)}$. Como resultado de la infección por el Helicobacter pylori, la mucosa gástrica sufre una serie de alteraciones histológicas que pueden progresar de gastritis crónica, a gastritis atrófica, metaplasia intestinal, displasia y carcinoma ${ }^{(10)}$. Esta serie de eventos (La Cascada de Correa) se asocia con un incremento significativo en el desarrollo de cáncer gástrico a cada estadio de la Cascada siendo significativo en la etapa de metaplasia intestinal cuando las lesiones histológicas causadas por el Helicobacter son más reacias de mejorar con tratamiento de la infección. Este "punto de no retorno" es muy importante para programas de monitoreo y detección temprana del cáncer gástrico ${ }^{(11)}$. El incremento de resistencia antibiótica del Helicobacter pylori a las medicinas actuales complican mucho más el pronóstico de esta infección ${ }^{(12,13)}$.

Desde el punto de vista quirúrgico, se indicó que entre 1990 y 2010, médicos del INEN evaluaron 9753 
pacientes con carcinoma gástrico y encontraron que $2402(25 \%)$ eran resecables, 1802 (18\%) irresecables, $5549(57 \%)$ inoperables y $222(2 \%)$ fueron excluidos por razones médicas. De los resecables, el 53\% requirieron gastrectomía total que generalmente incluye cirugía tipo D2 con disección ganglionar extensa que da resultados favorables en comparación con cirugías menos extensas de acuerdo a investigadores de $\mathrm{Asia}^{(14)}$. La supervivencia a los 5 años fue de 50\%, siendo de más del $80 \%$ para aquellos con estadios $1 \mathrm{~A}$ y IB, siendo menos del $20 \%$ en pacientes con estadios IIIB y IIIC. La supervivencia a los 10 años fue de $41.8 \%$.

La posible recurrencia local y sistémica de muchos pacientes operados con estadios II y III han estimulado la investigación de regímenes de quimioterapia y de quimio-radioterapia adyuvante ${ }^{(15,16)}$. Los resultados de estos estudios han sido modestos aunque el régimen de quimio-radioterapia de Macdonald et al. mejoró la supervivencia a los tres años a $50 \%$ vs $41 \%$ de cirugía sola ${ }^{(15)}$; sin embargo, el régimen fue considerado tóxico. Varios regímenes de quimioterapia perioperatoria han dado resultados de

Referencias bibliograficas

1. International Agency for Research on Cancer; World Health Organization; GLOBOCAN 2020.

2. Salazar MR, Regalado-Rafael R, Navarro JM, Montanez DM, Abugattas JE, Vidaurre T. El Instituto Nacional de Enfermedades Neoplásicas en el control del cáncer en el Perú. Rev Peru Med Exp Salud Pública. 2013;30(1):105-112.

3. Rodríguez RL, Yurgaky JS, Otero W, Faizal M. A Review of Paraneoplastic Syndromes in Gastrointestinal Tumors. Rev Colomb Gastroenterol / 2017;32(3). DOI https://doi.org/10.22516/25007440.155

4. Hu B, El Haji N, Sittler S, Lammert N, Barnes R, Meloni-Ehrig A. Gastric cancer: Classification, histology and application of molecular pathology; J Gastrointest Oncol 2012;3(3):251-261

5. Bang YJ, Cutsem EV, Feyereislova A, Chung HC, Shen L, Sawaki A, et al. Trastuzumab in combination with chemotherapy versus chemotherapy alone for treatment of HER2-positive advanced gastric or gastro-oesophageal junction cancer (ToGA): a phase 3, open-label, randomised controlled trial. The Lancet 2010:376:687-697.

6. Le DT, Uram JN, Wang H, Kemberling H, Eyring A, Bartlett B, et al. PD-1 blockade in mismatch repair deficient non-colorectal gastrointestinal cancers. J Clin Oncol 2016:34:195(Abstract).

7. Jaw-Town Lin. Screening of Gastric Cancer: Who, When, and How. Clinical Gastroenterology and Hepatology 2014;12:135-138.

8. Crew KD, Neugut AI. Epidemiology of gastric cancer. World J Gastroentero 2006; 12:354-362.

9. Fock KM, Talley N, Moayyedi P, Hunt R, Azuma T, Sugano K, et al. Asia-Pacific consensus guidelines on gastric cancer prevention. J Gastroenterol Hepatol sobrevida alentadores como el estudio FLOT 4 que incluye Epirubicin, Cisplatin, Fluorouracilo/Capecitabine o Docetaxel, Oxaliplatino, Leucovorin y Fluorouracilo $^{(16)}$. Este régimen es tóxico y se aconseja ser considerado en pacientes con buen estado funcional. En enfermedad metastásica, la administración de regímenes que contienen terapia anti Her - 2 neu a pacientes con sobreexpresión de este marcador da una ventaja de sobrevida media de 13.8 vs 11.1 meses $^{(5)}$. Estudios recientes investigan la eficacia de inmunoterapia en pacientes con expresión de anti PDl-1 sin resultados concluyentes todavía ${ }^{(6)}$.

En resumen, este Simposio enfocó al cáncer gástrico desde el punto de vista multidisciplinario. Se resaltó las limitaciones de nuestras políticas de salud y de seguro de salud que no cubren el monitoreo adecuado de pacientes con lesiones premalignas debidas al Helicobacter pylori. Se anotó las limitaciones en la prevención de la transmisión de la infección por el Helicobacter pylori. El desarrollo de resistencia antibiótica al tratamiento del Helicobacter pylori es de gran preocupación ya que ello facilita la evolución de lesiones premalignas a cáncer gástrico.

\section{Manuel Valdivieso Rodriguez, M.D. \\ Director de Educación, Peruvian American Medical Society (PAMS), Profesor Adjunto, Departamento de Medicina Interna, Alcance Global, Universidad de Michigan, Ann Arbor, Michigan.}

2008:23:351-365

0. Correa P, Piazuelo MB. The gastric precancerous cascade. J Dig Dis. 2012;13(1):2-9. 11. Koulis A, Buckle A, Boussioutas A. Premalignant lesions and gastric cancer: Current understanding. World J Gastrointest Oncol 2019;11(9):665-678.

12. Boehnke K, Valdivieso M, Bussalleu A, Sexton R, Thompson KC, Osorio S, Novoa Reyes, I, Crowley J, Baker LH, Xi C. Antibiotic resistance among Helicobacter pylor clinical isolates in Lima, Peru (SWOG Clinical Trial 1119). Infection and Drug Resistance; 2017:10:85-90.

13. Khien VV, Thang DM, Hai TM, Duat NQ, Khanh PH, Ha DT, et al. Management of Antibiotic-Resistant Helicobacter pylori Infection: Perspectives from Vietnam. Gut and Liver, 2019;13(5):483-497.

14. Tamura S, Takeno A, Miki H. Lymph node Dissection in Curative Gastrectomy for Advanced Gastric Cancer; International Journal of Surgical Oncology, 2011; Article 748745; doi: 10.1155/2011/748745.

15. Macdonald JS, Smalley SR, Benedetti, J, Hundahl SA, Estes NC, Stemmerman GN, et al. Chemoradioterapy after surgery compared with surgery alone for adenocarcinoma of the stomach or gastroesophageal junction. N Engl J Med 2001;345:725-730.

16. Al-Batran SE, Homann N, Pauligk C, Goetze TO, Meiler J, Kasper S, et al. On behalf of the FLOT4-AIO Investigators. Perioperative chemotherapy with fluorouracil plus leucovorin, oxaliplatin, and docetaxel versus fluorouracil or capecitabine plus cisplatin and epirubicin for locally advanced, resectable gastric or gastro-oesophageal junction adenocarcinoma (FLOT4): a randomised, phase 2/3 trial. The Lancet 2019;393:1948-1957.

Citar como: Valdivieso Rodríguez M. Simposio Cáncer Gástrico. Diagnóstico (Lima). 2021;60(2):69-70.

DOI: 10.33734 /diagnostico.v60i2.282

Correspondencia: Manuel Valdivieso Rodríguez. Correo electrónico: m.valdivieso.md@outlook.com 